\title{
Histological Changes in Lung Induced by Single Intratracheal Instillation on Bleomycin in Wistar Rats
}

\author{
Ramesh Verma ${ }^{1}$, Poonam Mehta ${ }^{1}$, Vishvesh Dalal' ${ }^{1}$, Manish V Patel ${ }^{1}$, \\ Parnav Buch ${ }^{2}$, Pratyush Patankar ${ }^{2}$ and Suresh Balakrishnan ${ }^{2 *}$ \\ ${ }^{1}$ Department of Toxicology, Jai Research Foundation, Valvada, Gujarat, India \\ ${ }^{2}$ Division of Toxicology, Department of Zoology, Faculty of Science, The M.S. \\ University of Baroda, Vadodara, Gujarat, India \\ *Corresponding Author: Suresh Balakrishnan, Division of Toxicology, Department \\ of Zoology, Faculty of Science, The M.S. University of Baroda, Vadodara, Gujarat, \\ India.
}

\begin{abstract}
The present study examined the histological changes in lung tissue induced by single intratracheal instillation on bleomycin in Wistar rats. Animals were treated with bleomycin at the dose of $6.5 \mathrm{U} / \mathrm{kg}$ body weight via., single intra-tracheal instillation. Histopathological abnormalities in lungs were assessed on day 21 using hematoxylin and eosin staining and Masson's trichrome staining. Single intra-tracheal instillation of bleomycin led to the extensive deposition of collagen in peribronchial and perialveolar region, which obliterated the alveolar spaces. Severe lung alveolitis and injury, which was manifested with interalveolar inflammation, thickening alveolar wall, bronchial erosion, and large fibrotic area inconsistently. The results of the present study speculate that inflammation is the forerunner of fibrosis induced by single intra-tracheal instillation of bleomycin.

Keywords: Bleomycin; Lung Fibrosis
\end{abstract}

\section{Introduction}

Idiopathic pulmonary fibrosis is a chronic progressive and ultimately fatal lung disease and poor response to available medical therapy. Its prediction is poor and the outcome even worse than in many malignant diseases. Idiopathic pulmonary fibrosis is one of the most common interstitial lung diseases and is characterized by the histological pattern of usual interstitial pneumonia [3].

The natural history of pulmonary fibrosis is unknown, and the onset of symptoms is gradual, usually starting with a non-productive cough. With the involvement of larger areas of the lung, severe dyspnea at rest, and signs of right heart failure also develop [4]. In some cases, the clinical state is preserved for several years, but the majority of patients deteriorate more rapidly. Mortality during an acute exacerbation is high. The prevalence of idiopathic pulmonary fibrosis is estimated at 20/100,000 for males and 13/100,000 for females, and survival time from diagnosis ranges from 2 to 4 years [10].

Histological characteristics include remodeling of lung architecture with fibroblastic foci and "honeycombing". The lung involvement is patchy with a predominantly basal and subpleural pattern of matrix deposition and tissue distortion within the pulmonary interstitium, leading to impaired gas transfer and respiratory failure $[4,17]$. Most patients present at a later stage of the disease.
Treatment options for pulmonary fibrosis are limited. The clinical management focuses on the treatment of complications, supportive care, and in a few cases, involves lung transplantation. Antiinflammatory drugs such as prednisone may carry symptomatic relief. Still, they do not appear to halt the progression of fibrosis, and their beneficial effects in idiopathic pulmonary fibrosis remain in question. Cytotoxic drugs have not been shown to improve lung function or life expectancy and may be associated with harmful side effects [11].

Animal models play an important role in the investigation of diseases, and many models are established to examine pulmonary pathobiology. So far, the standard agent for the induction of experimental pulmonary fibrosis in animals is bleomycin, so the same was used as the animal model in the present study. Bleomycin, first discovered in 1962, is a glycosylated linear non-ribosomal peptide chemotherapeutic antibiotic produced by the bacterium "Streptomyces verticillus" [1,14]. Its use in animal models of pulmonary fibrosis is based on the fact that fibrosis is one of the major adverse side effects of bleomycin in human cancer therapy. Bleomycin plays an important role in the treatment of lymphoma, squamous cell carcinomas, germ cell tumors, and malignant pleural effusion, where it is injected intrapleurally. It is believed that bleomycin acts by causing single and double-strand D.N.A. breaks in tumor cells and thereby interrupting the cell cycle. This happens by chelation 
of metal ions and reaction of the formed pseudoenzyme with oxygen, which leads to the production of DNA-cleaving superoxide and hydroxide free radicals [6]. Overproduction of reactive oxygen species can lead to an inflammatory response causing pulmonary toxicity, activation of fibroblasts, and subsequent fibrosis [5,7]. Bleomycin hydrolase, a bleomycin inactivating enzyme, critically influences the effects of this drug on different tissues. The lungs maintain low levels of the enzyme and therefore are more susceptible to bleomycin-induced tissue injury [12], so the same was used as the animal model in the present study.

\section{Materials and Methods}

Animals

Specific pathogen-free, healthy young adult male Wistar rats (RccHan: WIST) were used in this study. They were obtained from the barrier maintained rodent animal breeding facility, Jai Research Foundation, Vapi, India. All the animals were fed with standard Teklad certified Global high fiber rat feed manufactured by Harlan, U.S.A., and U.V. sterilized water filtered through Kent reverse osmosis water filtration system were provided ad libitum. The rats were kept in a controlled environment with an alternating cycle of 12-h light and dark. The animals used in this study were handled and treated in accordance with the strict guiding principles of the National Institutes of Health Guide for the Care and Use of Laboratory Animals and were approved by the Institutional Animal Ethics Committee Guidelines. Jai Research Foundation is also accredited with the Association for Assessment and Accreditation of Laboratory Animal Care (AAALAC) that promotes the humane treatment of animals in science.

\section{Experimental design}

The rats were anesthetized via ketamine $(80 \mathrm{mg} / \mathrm{kg}$ body weight, i.p.) and xylazine (20 mg/kg body weight, i.p.) anesthesia [13]. A midline incision was made in the neck, and the trachea was exposed. Later, with the help of a tracheal cannula under direct visualization, bleomycin was instilled. For the induction of pulmonary fibrosis, the rats received a single dose of $6.5 \mathrm{U} / \mathrm{kg}$ body weights, bleomycin sulfate by intratracheal instillation [16]. On day 21 , animals were sacrificed, and lungs were collected.

\section{Collagen specific studies}

Excess collagen deposition is the hallmark of pulmonary fibrosis. After sacrificed, lung tissue was fixed in $10 \%$ neutral buffer formalin and routinely processed and embedded in paraffin. Lung tissue collagen content was examined using Masson trichrome, which is specific staining for collagen.

\section{Histological studies}

After sacrificed, each lung tissue was perfused and fixed in 10\% neutral buffer formalin and routinely processed and embedded in paraffin. Serial sections were cut and stained with hematoxylin \& eosin for illuminate pathological changes associated with bleomycin-induced lung fibrosis.

\section{Materials}

Bleomycin hydrochloride was procured from the market and was in the form of bleomycin ampoules (15 units) manufactured by Biochem Pharmaceutical Industries Ltd. All other chemicals were of the highest grade commercially available.

\section{Results}

Histopathological abnormalities in lungs were assessed on day 21 using hematoxylin and eosin staining and Masson's trichrome staining.

\section{Masson's trichrome staining}

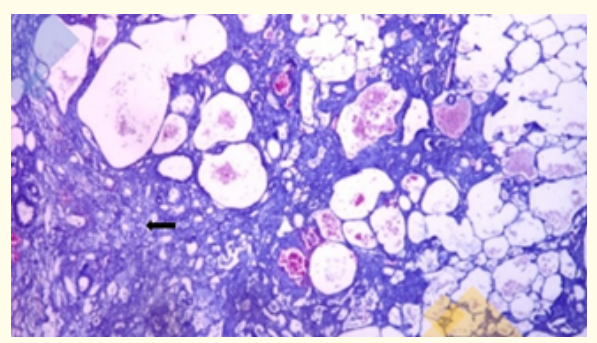

Figure 1

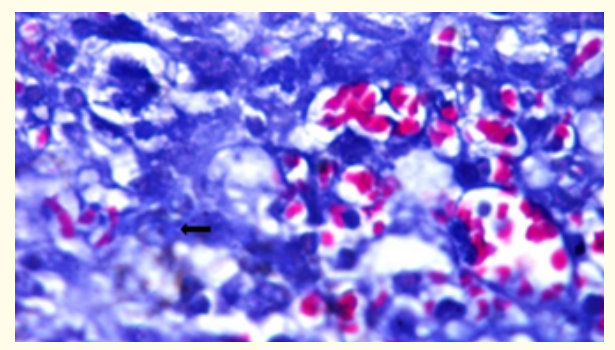

Figure 2

\section{Hematoxylin and eosin staining}

As shown in figure 1 and 2, single instillation of bleomycin resulted in severe lung alveolitis and injury, which was manifested with interalveolar inflammation, thickening alveolar wall, bronchial erosion and large fibrotic area inconsistently. Severe lung injury manifested as congestion and edema of interalveolar spaces, interalveolar inflammation, thickened alveolar wall, collapsed alveoli with bronchial erosion, and patchy cheesy fibrotic areas.

\section{Discussion and Conclusion}

Animal models do not completely imitate the interstitial lung disease of humans and usually require an acute lung injury response followed by a slower fibrosis phase that differs from the chronic and insidious features of this disease. Inflammation is a major component in the pathogenesis of this lung disease that is orchestrated in part by endogenous and migrating leukocytes. These leukocytes, together with lung epithelial and endothelial cells, create a feedback loop where stimuli from injury responses, can activate alveolar and interstitial macrophages [8]. 
The clinical worth of bleomycin, an anti-cancer drug, has been hampered due to its detrimental effects. The major side-effect of this drug is the induction of lung fibrosis in patients treated with bleomycin. The bleomycin animal model of lung fibrosis is an established and widely used model of human lung fibrosis. There have been a number of studies employing bleomycin in different animal models. The use of these animal models has helped in partly establishing the pathways of lung damage leading to fibrosis and by comparison studies of patients with lung pneumopathy, have validated many of these animal studies [15].

In the current study, we have used a Wistar rat model of lung fibrosis created by challenging the rats with a single dose of bleomycin sulfate by intratracheal instillation. Single instillation of bleomycin causes destruction of the lung architecture, leading to pulmonary fibrosis that was characterized by an increase in collagen deposition in the lungs.

Moreover, histopathological observation showed an abnormal histological profile of the lung tissue in bleomycin-administered rats. Notable deviant histoarchitecture of the lung from the drugtreated animals includes collapsed alveolae, thickened alveolar wall, and abnormal collagen deposition.

Since inflammation is the forerunner of fibrosis, controlling the degree of inflammation could minimize the release of various inflammatory mediators known to be involved in collagen synthesis and accumulation, and thus attenuating the development of bleomycin-induced lung fibrosis [2,9].

\section{Conflict of Interest Statement}

The authors declare that there are no conflicts of interest.

\section{Acknowledgments}

The authors are very grateful to Dr A. Deshpande for his kind support for providing the facility to conducting the study in the experimental premises. This work was financially supported by Jai Research Foundation, Vapi, India.

\section{Bibliography}

1. Sin JC., et al. "Degrading Endocrine disrupting chemicals from wastewater by photocatalysis: A review". International Journal of Photoenergy (2012): 185159.

2. Nakata $\mathrm{K}$ and Fujishima A. "TiO 2 photocatalysis: Design and applications". Journal of Photochemistry and Photobiology C: Photochemistry Review 13.3 (2012): 169-189.

3. Vilar VJ., et al. "Solar photocatalysis of a recalcitrant coloured effluent from a wastewater treatment plant". Photochemical and Photobiological Sciences 8.5 (2009): 691-698.
4. Fujishima A., et al. "Photosensitized electrolytic oxidation on semiconducting n-type $\mathrm{TiO}_{2}$ electrode". The Journal of the Society of Chemical Industry, Japan 72 (1969): 108-113.

5. Xie H., et al. "Photodegradation of benzene by $\mathrm{TiO}_{2}$ nanoparticles prepared by flame CVD process". Particuology 9.1 (2011): 75-79.

6. Ameta A., et al. "Photocatalytic degradation of methylene blue by nanosized vanadium doped $\mathrm{TiO}_{2}$ loaded on zeolite". International Journal of Chemical Sciences 13.3 (2015): 1485-1494.

7. Haritha E., et al. "Green chemical approach towards the synthesis of SnO2 NPs in argument with photocatalytic degradation of diazo dye and its kinetic studies". Journal of Photochemistry and Photobiology B: Biology 162 (2016): 441-447.

8. Goharshadi E., et al. "Photocatalytic degradation of reactive black 5 azo dye by zinc sulfide quantum dots prepared by a sonochemical method". Materials Science in Semiconductor Processing 16.4 (2013): 1109-1116.

9. Wang X., et al. "Large-scale synthesis well-dispersed ZnS microspheres and their photoluminescence, photocatalysis properties". Material Characterization 59.12 (2008): 1765-1770.

10. Chen F., et al. "Facile synthesis of $\mathrm{ZnS}$ nanoparticles and their excellent photocatalytic performance". Ceramics International 41.5A (2015): 6645-6652.

11. Chankhanittha T and Nanan S. "Hydrothermal synthesis, characterization and enhanced photocatalytic performance of $\mathrm{ZnO}$ toward degradation of organic azo dye". Material Letters 226 (2018): 79-82.

12. Raheem RA., et al. "Photocatalytic degradation of reactive green dye by using zinc oxide". Journal of Chemical and Pharmaceutical Sciences 9.3 (2016): 1134-1138.

13. Shi JW., et al. "Low-temperature synthesis of $\mathrm{CdS} / \mathrm{TiO}_{2}$ composite photocatalysts: Influence of synthetic procedure on photocatalytic activity under visible light". Journal of Molecular Catalysis A: Chemical 356 (2012): 53-60.

14. Kostova N and Dutkova E. "Mechanochemical synthesis and properties of $\mathrm{ZnS} / \mathrm{TiO}^{2}$ composites". Bulgarian Chemical Communications 48 (2016): 161-166.

15. Upadhyay R., et al. "Photocatalytic degradation of azure B using Copper Hexacyanoferrate (II) as semiconductor". International Journal of Chemical Sciences 11.1 (2013): 331-340. 
16. Parveen $\mathrm{K}$ and Vyas K. "Photocatalytic degradation of Rose Bengal by Ni-doped titanium dioxide". Acta Chimica and Pharmaceutica Indica 6.4 (2016): 135-143.

17. Sharma S., et al. "Use of semi-conducting bismuth sulfide as a photocatalyst for degradation of rose Bengal". Macedonian Journal of Chemistry and Chemical Engineering 30.2 (2011): 229-234.

\section{Assets from publication with us}

- Prompt Acknowledgement after receiving the article

- Thorough Double blinded peer review

- Rapid Publication

- Issue of Publication Certificate

- High visibility of your Published work

Website: www.actascientific.com/

Submit Article: www.actascientific.com/submission.php

Email us: editor@actascientific.com

Contact us: +919182824667 